\title{
Procedimiento Metodológico para la Ponderación Relativa de la Importancia Ambiental de las Acciones de Proyectos
}

\section{Methodological Procedure for the Relative Weighting of the Environmental Importance of Project Actions}

Presentación: 03/06/2019

Aprobación: 19/09/2019

\section{Susana LLAMAS}

Facultad de Ingeniería, Universidad Nacional de Cuyo, Mendoza - Argentina sllamas@uncuyo.edu.ar

\section{Jorge BARON}

Facultad de Ingeniería, Universidad Nacional de Cuyo, Mendoza - Argentina jbaron@uncuyo.edu.ar

\section{Resumen}

Se presenta un procedimiento metodológico, complementario a la Evaluación de Impacto Ambiental (EIA), que define una estructura jerárquica para el proyecto, permite calcular el peso relativo de cada acción y comparar analíticamente diferentes alternativas. Se asignaron 1000 Unidades de Importancia de Acciones de Proyecto (UIAP) al proyecto en estudio para distribuirlas entre sus elementos, se utilizó decisión multicriterio para calcular el conjunto de coeficientes $\left(p_{j}\right)$ que representa el peso relativo de cada acción. Con la aplicación del procedimiento desarrollado a la EIA de un proyecto minero se comprobó la validez del método desarrollado para justificar matemáticamente la alternativa seleccionada.

Palabras clave: acciones de proyectos, análisis de alternativas, impacto ambiental, multicriterio 


\begin{abstract}
A complementary methodological procedure to the Environmental Impact Assessment (EIA) that defines a hierarchical structure for the project, allows calculate the relative weight of each action and analytically compares different alternatives is presented. 1000 Units of Importance of Project Actions (UIPA) were assigned to the project under study to be distributed among its elements. Multicriteria decision was used to calculate the set of coefficients $\left(p_{j}\right)$ that represents the relative weight of each action. With the application of the procedure developed to the EIA of a mining project, the validity of the method developed to justify mathematically the selected alternative was verified.
\end{abstract}

Keywords: analysis of alternatives, environmental impact, multi-criteria, project actions

\title{
Introducción
}

La finalidad de la Evaluación de Impacto Ambiental (EIA) es determinar los efectos que las acciones de un proyecto podrían ocasionar sobre la calidad ambiental de los factores susceptibles de resultar afectados por su ejecución. Entre las diferentes metodologías desarrolladas para la elaboración de una EIA se encuentran las siguientes: ad hoc (Dee et al. 1972), listas de chequeo (Canter 1982, Canter and Canty 1993), matrices causa-efecto (Leopold et al. 1971, Thompson 1990, Conesa Fdez.-Vítora 1993, Goyal and Deshpande 2001, Gómez Orea 2010, Ijäs et al. 2010), diagramas de red (Peterson et al. 1974, Perdicoúlis and Glasson 2006), métodos multicriterio (Huang et al. 1995, Ramanathan 2001, Leknes 2001, Steinemann 2001, Jansen 2001, Zhao et al. 2006, Hoyos 2010, Lin et al. 2010, Huang et al. 2011, Kaya and Kahraman 2011, Toro et al. 2013, Laivina and Pubule 2014), técnicas difusas (Parashar et al. 1997, Enea and Salemi 2001, Siqueira Campos Boclin and de Melo 2006, Cloquell-Ballester et al. 2007, Liu and Lai 2009, Blanco Morón et al. 2009, Peche and Rodríguez 2011, Yang et al. 2011, Cardenas et al. 2016) y sistemas cartográficos (Warner and Preston 1974, van der Werf et al. 2007).

El método matricial de EIA es uno de los más empleados porque permite organizar la información en filas y columnas. En las filas ( $)$ se presenta el ambiente desagregado en subsistemas, componentes y factores (Dee et al. 1972); en las columnas (i) se colocan las acciones del proyecto (Garmendía et al. 2005, Conesa Fdez.-Vítora 2010, Gómez Orea 2010). Una vez concluidas las etapas iniciales de descripción del proyecto y caracterización del ambiente, la EIA continúa con la identificación de las interacciones $\left(I_{i j}\right)$ entre las acciones del proyecto y los factores ambientales y prosigue con la determinación de la importancia y la magnitud de los impactos ambientales, que se expresan en función de la importancia relativa de los factores ambientales.

Para la ponderación relativa de los elementos del ambiente se utiliza el EES (Environmental Evaluation System) que distribuye 1000 PIU (Parameter Importance Units) en una estructura jerárquica de cuatro niveles compuesta por sistemas, subsistemas, componentes y factores ambientales (Dee et al. 1972). Las PIU se utilizan luego para determinar la importancia 
relativa de los impactos ambientales sobre los factores estudiados.

Cuando se confeccionan las matrices de EIA todas las acciones del proyecto participan del procedimiento con el mismo peso relativo. Esto implica que en la descripción del proyecto no se tiene en cuenta la importancia relativa de las acciones que tienen lugar en las diferentes etapas de su desarrollo, ni los diferentes tipos de obras o las actividades necesarias para su ejecución.

En la EIA se incluye una sección final con el plan de gestión ambiental que contiene dos programas específicos; el programa de vigilancia y control ambiental con las medidas preventivas propuestas para eliminar o reducir los efectos ambientales de las acciones del proyecto y el programa de monitoreo ambiental con las medidas correctivas, mitigadoras y compensadoras que se implementan sobre el factor ambiental afectado por las acciones del proyecto cuyos efectos no se pueden eliminar o reducir.

Sin embargo, el proponente del proyecto solo puede ejercer algún grado de control sobre la alternativa de proyecto seleccionada para que las etapas, las obras, las actividades y las acciones se realicen según el plan de ejecución programado, con el personal, los materiales, los equipos, los insumos, los residuos y los recursos financieros declarados en la EIA.

El procedimiento metodológico que se describe en las siguientes secciones, es complementario a la EIA y organiza los elementos del proyecto en una estructura jerárquica compuesta por cinco niveles, asigna 1000 Unidades de Importancia de Acciones de Proyecto (UIAP) al proyecto en estudio y emplea el proceso analítico jerárquico (AHP: Analytic Hierarchy Process) (Saaty 1977, Saaty 1978, Saaty 1987, Saaty 2008), para obtener un conjunto de coeficientes ( $p_{-} \mathrm{j}$ ) que representa el peso relativo de cada acción en el proyecto y queda expresado por medio de un indicador denominado Unidades de Importancia de Acciones de Proyecto (UIAP).

En la matriz de EIA las UIAP (Unidades de Importancia de Acciones de Proyecto) se ubican en las columnas ( $i$ ), las PIU (Parameter Importance Units) en las filas ( $j$ ) y, utilizando las expresiones de cálculo desarrolladas para obtener el valor relativo del impacto ambiental causado por las acciones del proyecto $\left(I_{R i}\right)$ y el valor relativo del impacto ambiental recibido por los factores $\left(I_{R j}\right)$ se analizan comparativamente las alternativas de proyecto consideradas. Finalmente se confecciona el programa de vigilancia y control con las medidas preventivas para las acciones más importantes y se prepara un programa de monitoreo para el seguimiento de los factores ambientales más significativos.

\section{Desarrollo}

El procedimiento metodológico, compuesto por los pasos que se explican a continuación, se desarrolló para resolver el problema de la falta de ponderación de las acciones de los proyectos sometidos al procedimiento de EIA.

Para demostrar su simplicidad todos los cálculos se realizaron con planillas de cálculo Excel $^{\circledR}$ de Microsoft Office ${ }^{\circledR}$.

\section{Estructura jerárquica del proyecto}

Para la representación de los elementos del proyecto se definió una estructura jerárquica compuesta por los cinco niveles que se presentan en la Figura 1. 


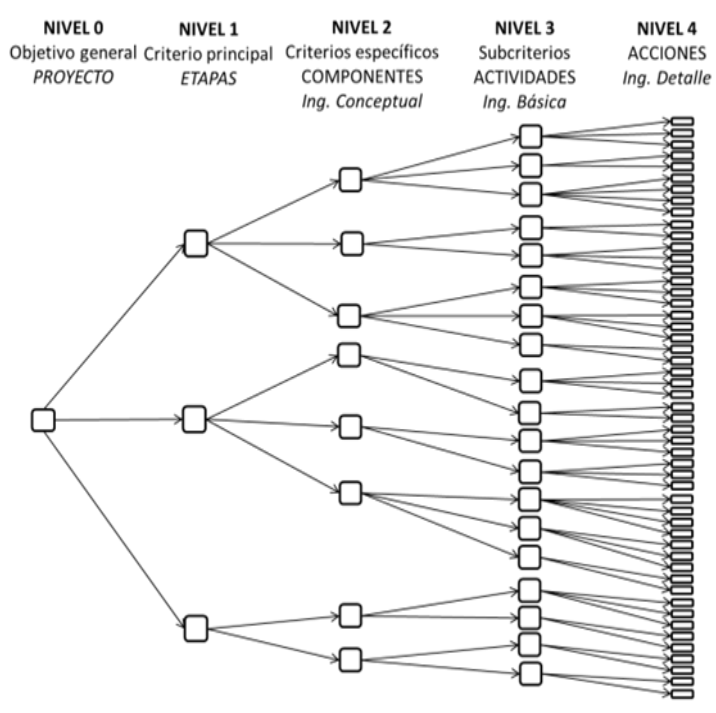

Figura 1. Esquema de la estructura jerárquica del proyecto. Elaboración propia

El nivel 0 corresponde al objetivo general de análisis, que es el proyecto en estudio. La descripción del proyecto y sus acciones se realiza para las etapas de construcción, funcionamiento y cierre, por lo que las tres etapas del proyecto constituyen los criterios principales y ocupan el nivel 1 de la jerarquía. En el nivel 2 se ubican los componentes, que son todas las obras complementarias subordinadas a las etapas del proyecto principal obtenidas de la Ingeniería conceptual, por ejemplo: Obras civiles. Obras hidráulicas. Obras industriales. Obras de mantenimiento, Obras de desmantelamiento, u otras. En el nivel 3 se incluyen las actividades, que son las subdivisiones de los componentes del proyecto; cada actividad tiene un objetivo y una planificación preestablecidos y debe cumplir las especificaciones de cantidad, calidad, recursos requeridos, materiales necesarios, personal a emplear y tiempos de ejecución (Hillier and Liebermann 2010, Taha 2006). El nivel 4 contiene las acciones del proyecto, definidas a partir de las actividades identificadas en el nivel precedente y determinadas durante el desarrollo de la Ingeniería de detalle.

\section{Unidades de Importancia de Acciones de Proyecto (UIAP)}

Se asignaron 1000 UIAP al proyecto en estudio, ubicado en el nivel 0 de la estructura jerárquica de la Figura 1. Para obtener los coeficientes $\left(p_{j}\right)$, que representan el peso relativo de cada elemento del proyecto, las 1000 UIAP se distribuyeron entre los niveles de la jerarquía empleando el proceso analítico jerárquico (AHP: Analytic Hierarchy Process) (Saaty 1977, Saaty 1978, Saaty, 1987, Saaty 2008) que utiliza matrices de comparaciones por pares de criterios y permite descomponer estructuras complejas en sus componentes, ordenarlos jerárquicamente, obtener valores numéricos para los juicios de preferencia comparando pares de elementos y sintetizarlos para determinar qué variable tiene la prioridad más alta (Saaty 1987, p. 166). 
Las valoraciones de los juicios de preferencia se obtienen utilizando el método Delphi de convergencia (Landeta 1999), que se basa en la realización de encuestas a paneles de expertos extraídos de los grupos sociales con interés en el proyecto (Conesa Fdez.-Vítora 2010, p. 261-263).

Las matrices de comparaciones por pares de criterios deben cumplir con las condiciones algebraicas de ser cuadradas: $\mathrm{A}=\left[a_{i j}\right] ; \forall i, j=1,2, \ldots, k$, positivas: $a_{i j}>0 ; \forall i, j=1,2, \ldots, k$, recíprocas: $a_{i j} \cdot a_{j i}=1 ; \forall i, j=1,2, \ldots, k$, con elementos diagonales iguales a uno: $a_{i j}=1, ; i, j=1,2, \ldots, k$ y consistentes: $a_{i j}=a_{i k} \cdot a_{k j}, \forall i, k, j$.

Cuando se logra la consistencia en las valoraciones de los juicios de preferencia, la matriz tiene un rango unitario y es suficiente conocer una fila de la matriz para construir las entradas restantes. Es decir que si se conoce la primera fila, entonces $a_{i j}=a_{1 j} / a_{1 i}$ (bajo la suposición que $a_{1 i} \neq 0$ para todo $\left.i\right)$. La expresión $\left(\lambda_{\max }-n\right) /(n-1)$ mide la consistencia o confiabilidad de la información por parte de un individuo para que tenga la forma $a_{i} / a_{j}$ (Saaty 1977, p. 245).

El AHP utiliza la escala fundamental de números absolutos propuesta por Saaty (1977, p. 246) cuyos valores son los de la Tabla 1 y representan la fuerza de cada preferencia.

\begin{tabular}{|c|c|c|}
\hline $\begin{array}{l}\text { Intensidad de impor- } \\
\text { tancia en una escala } \\
\text { absoluta }\end{array}$ & Definición & Explicación \\
\hline 1 & Igual importancia & Dos actividades contribuyen igual al objetivo \\
\hline 3 & $\begin{array}{l}\text { Importancia moderada de } \\
\text { uno respecto del otro }\end{array}$ & $\begin{array}{l}\text { La experiencia y el juicio favorecen una activ- } \\
\text { idad sobre otra }\end{array}$ \\
\hline 5 & Importancia esencial o fuerte & $\begin{array}{l}\text { La experiencia y el juicio favorecen más una } \\
\text { actividad sobre otra }\end{array}$ \\
\hline 7 & Importancia muy fuerte & $\begin{array}{l}\text { Una actividad está fuertemente favorecida y } \\
\text { su dominio demostrado en la práctica }\end{array}$ \\
\hline 9 & Importancia extrema & $\begin{array}{l}\text { La evidencia a favor de una actividad sobre } \\
\text { otra corresponde al mayor orden posible de } \\
\text { afirmación }\end{array}$ \\
\hline $2,4,6,8$ & $\begin{array}{l}\text { Valores intermedios entre las } \\
\text { dos sentencias adyacentes }\end{array}$ & $\begin{array}{l}\text { Cuando se necesita compromiso entre los } \\
\text { valores anteriores }\end{array}$ \\
\hline Recíprocas & $\begin{array}{l}\text { Si la actividad i tiene uno de } \\
\text { los números anteriores que } \\
\text { se le asignen en comparación } \\
\text { con la actividad j, entonces } \\
\text { j tiene el valor inverso } \\
\text { en comparación con i }\end{array}$ & \\
\hline Racionales & Ratios derivados de la escala & $\begin{array}{l}\text { Si la consistencia tuviese que ser forzada por } \\
\text { la obtención de } n \text { valores numéricos para } \\
\text { abarcar la matriz }\end{array}$ \\
\hline
\end{tabular}

Tabla 1. Escala fundamental de preferencias. Fuente: Saaty 1977, p. 246

La consistencia de cada matriz de comparaciones por pares de criterios de orden $\mathrm{n}$ se comprueba obteniendo el principal valor propio $\left(\lambda_{\max }\right)$, con el cual se calcula el índice de consistencia aleatoria $I C=\left(\lambda_{\max }-\mathrm{n}\right) /(\mathrm{n}-1)$, luego se obtiene la relación de consistencia 
$R C=I C /$ (Índice aleatorio), donde el Índice aleatorio depende del tamaño (n) de la matriz de comparaciones por pares cuyos valores se presentan en la Tabla 2. Finalmente se verifica que el IC resulte inferior al $10 \%$ recomendado por el autor del método (Saaty 1977, p. 244251, Saaty 1987, p. 172).

\begin{tabular}{|c|c|c|c|c|c|c|c|c|c|c|}
\hline $\mathbf{n}$ & 1 & 2 & 3 & 4 & 5 & 6 & 7 & 8 & 9 & 10 \\
\hline $\begin{array}{c}\text { Índice } \\
\text { aleatorio }\end{array}$ & 0 & 0 & 0.58 & 0.90 & 1.12 & 1.24 & 1.32 & 1.41 & 1.45 & 1.49 \\
\hline
\end{tabular}

Tabla 2. Índice promedio de consistencia aleatorio. Fuente: Saaty 1977, p. 244-251, Saaty 1987, p. 171

Utilizando el método Delphi de convergencia (Landeta 1999), se realizó una distribución preliminar de las 1000 UIAP entre los criterios principales del nivel 1, en correspondencia con las etapas de construcción, funcionamiento y cierre. Se preparó una matriz cuadrada de orden $n=3$ con los valores preliminares asignados a los criterios principales del nivel 1 para obtener una valoración cuantificada $\left(C_{i j}\right)$ inicial de los pares $c_{i}, c_{j}$ y definir preferencias según los cocientes $\left(c_{i} / c_{j}\right) \forall i, j=1,2,3$.

\section{Resultados}

El procedimiento metodológico desarrollado para representar la estructura jerárquica de un proyecto, calcular los coeficientes $\left(p_{j}\right)$ de cada elemento y obtener las UIAP se presenta aplicado a la fase de desarrollo de la Ingeniería básica de un proyecto para la extracción y procesamiento de minerales metalíferos, con algunas modificaciones menores para proteger la confidencialidad de la información técnica elaborada por el proponente del proyecto y preservar los derechos de propiedad intelectual de los autores de la EIA.

El ejemplo de aplicación consiste en la EIA de un proyecto para la explotación de un yacimiento minero metalífero, desarrollado a nivel de Ingeniería básica, situado en el sector central de la cordillera de Los Andes, en la provincia de Mendoza, República Argentina.

El tiempo estimado para la terminación de las obras de construcción de la totalidad de las instalaciones industriales necesarias para el procesamiento del mineral extraído es de 20 meses.

Para la explotación de la mina se adoptó el método de operación a cielo abierto que se completará en 10 años y para la etapa de cierre se estimó una duración de 12 meses.

Se trata de una operación minera con perforación convencional que involucra tareas de voladura con empleo de explosivos, carga del material en bruto y transporte en camiones hasta las instalaciones industriales para su procesamiento. El procesamiento industrial básico del mineral se realizará en cuatro etapas: 1) Molienda primaria. 2) Trituración y molienda fina. 3) Flotación. 4) Espesado y depósito de colas.

\section{Estructura jerárquica del proyecto}

Se utilizó la documentación de la Ingeniería básica del proyecto minero del ejemplo para preparar una lista con los elementos de cada nivel y organizarlos como se muestra en la Tabla 3. 


\begin{tabular}{|c|c|c|c|}
\hline ETAPA & COMPONENTES & ACTIVIDADES & ACCIONES \\
\hline \multirow{16}{*}{ 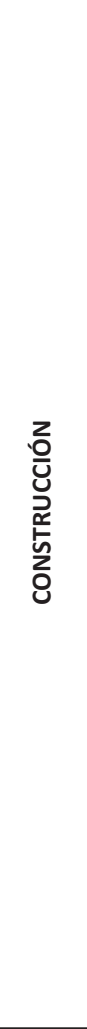 } & \multirow{11}{*}{ Componente 1: OBRAS CIVILES } & \multirow{3}{*}{$\begin{array}{l}\text { Actividad A: Accesos y caminos de circu- } \\
\text { lación interna. Canteras }\end{array}$} & Acción 1: Abrir caminos de acceso \\
\hline & & & Acción 2: Abrir caminos internos \\
\hline & & & Acción 3: Excavar canteras \\
\hline & & \multirow{2}{*}{$\begin{array}{l}\text { Actividad B: Construcción de edificios de } \\
\text { proceso y auxiliares }\end{array}$} & Acción 4: Construir campamento \\
\hline & & & $\begin{array}{l}\text { Acción 5: Construir plantas de proceso } \\
\text { y Oficinas }\end{array}$ \\
\hline & & $\begin{array}{l}\text { Actividad C: Acondicionamiento escom- } \\
\text { breras }\end{array}$ & $\begin{array}{l}\text { Acción 6: Acondicionar escombreras } \\
\text { de estéril }\end{array}$ \\
\hline & & Actividad D: Montaje plantas de proceso & Acción 7: Montar máquinas y equipos \\
\hline & & $\begin{array}{l}\text { Actividad E: Construcción plantas de } \\
\text { tratamiento }\end{array}$ & $\begin{array}{l}\text { Acción 8: Construir plantas de tratami- } \\
\text { ento de Residuos + Efluentes }\end{array}$ \\
\hline & & $\begin{array}{l}\text { Actividad F: Acondicionamiento estacio- } \\
\text { namientos }\end{array}$ & Acción 9: Nivelar y compactar \\
\hline & & \multirow{2}{*}{ Actividad G: Traslados } & Acción 10: Desplazar trabajadores \\
\hline & & & Acción 11: Transportar materiales \\
\hline & \multirow{2}{*}{ Componente 2: OBRAS HIDRÁULICAS } & \multirow{2}{*}{$\begin{array}{l}\text { Actividad H: Construcción obras de captac- } \\
\text { ión y distribución de agua }\end{array}$} & Acción 12: Construir toma de agua \\
\hline & & & Acción 13: Distribuir agua \\
\hline & \multirow{2}{*}{ Componente 3: OBRAS ELÉCTRICAS } & $\begin{array}{l}\text { Actividad I: Montaje subestación trans- } \\
\text { formadora }\end{array}$ & $\begin{array}{l}\text { Acción 14: Montar subestación trans- } \\
\text { formadora }\end{array}$ \\
\hline & & $\begin{array}{l}\text { Actividad J: Montaje líneas de distribución } \\
\text { de energía eléctrica }\end{array}$ & Acción 15: Tender líneas eléctricas \\
\hline & Componente 4: OBRAS INDUSTRIALES & $\begin{array}{l}\text { Actividad K: Instalación de máquinas y } \\
\text { equipos }\end{array}$ & Acción 16: Instalar máquinas y equipos \\
\hline \multirow{11}{*}{ 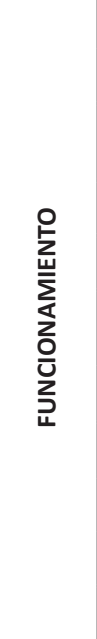 } & \multirow{2}{*}{ Componente 5: APERTURA MINA } & Actividad L: Excavación a cielo abierto & Acción 17: Excavar \\
\hline & & Actividad $\mathbf{M}$ : Explosiones & Acción 18: Detonar \\
\hline & $\begin{array}{l}\text { Componente 6: TRASLADO MINERAL } \\
\text { EN CAMIÓN }\end{array}$ & $\begin{array}{l}\text { Actividad N: Movimiento de minerales } \\
\text { desde la mina hasta fase trituración }\end{array}$ & Acción 19: Mover mineral en camiones \\
\hline & Componente 7: TRITURADORAS & Actividad O: Reducción de tamaño & Acción 20: Triturar \\
\hline & Componente 8: ZARANDAS & Actividad P: Separación por tamaño & Acción 21: Separar con zarandas \\
\hline & Componente 9: ACOPIO MINERAL & Actividad Q: Alimentación por cinta & $\begin{array}{l}\text { Acción 22: Transportar minerales por } \\
\text { cintas }\end{array}$ \\
\hline & Componente 10: MOLINO & Actividad R: Molienda & Acción 23: Moler-molino de bolas \\
\hline & Componente 11: CELDAS FLOTACIÓN & Actividad S: Flotación & Acción 24: Separar por densidad \\
\hline & \multirow{2}{*}{$\begin{array}{l}\text { Componente 12: ESPESADO Y FIL- } \\
\text { TRADO }\end{array}$} & \multirow{2}{*}{ Actividad T: Concentración } & Acción 25: Espesar \\
\hline & & & Acción 26: Filtrar \\
\hline & Componente 13: DEPÓSITO DE COLAS & Actividad U: Despacho de colas & Acción 27: Enviar a depósito de colas \\
\hline \multirow{5}{*}{ 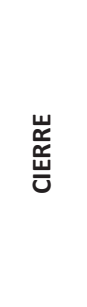 } & \multirow{2}{*}{ Componente 14: DEPÓSITO DE COLAS } & \multirow[t]{2}{*}{ Actividad V: Cierre y clausura } & $\begin{array}{l}\text { Acción 28: Cerrar operaciones depósito } \\
\text { de colas }\end{array}$ \\
\hline & & & Acción 29: Clausurar depósito de colas \\
\hline & \multirow{2}{*}{ Componente 15: ESCOMBRERAS } & \multirow{2}{*}{$\begin{array}{l}\text { Actividad W: Cierre y clausura escom- } \\
\text { breras }\end{array}$} & $\begin{array}{l}\text { Acción 30: Cerrar operaciones escom- } \\
\text { breras }\end{array}$ \\
\hline & & & Acción 31: Clausurar escombreras \\
\hline & Componente 16: MONITOREO & Actividad X: Monitoreo & Acción 32: Muestreo y análisis \\
\hline
\end{tabular}

Tabla 3. Elementos incluidos en cada nivel de la estructura del proyecto. Elaboración propia 


\section{Unidades de Importancia de Acciones de Proyecto (UIAP)}

Utilizando el método Delphi de convergencia (Landeta 1999) para asignar los valores preliminares a los criterios principales del nivel 1 se preparó una matriz cuadrada de orden

$n=3$. La Tabla 4 presenta la asignación preliminar de las 1000 UIAP entre los tres criterios principales del nivel 1 para el proyecto del ejemplo, obtenida a partir de la duración de cada etapa del proyecto (Construcción: 20 meses. Funcionamiento: 10 años. Cierre: 12 meses) y con la información de la Ingeniería básica.

\begin{tabular}{|c|c|c|c|c|}
\hline \multirow{2}{*}{ NIVEL 1 } & & Construcción & Funcionamiento & Cierre \\
\cline { 2 - 5 } & UIAP & $\mathbf{3 0 0}$ & $\mathbf{5 0 0}$ & $\mathbf{2 0 0}$ \\
\hline Construcción & $\mathbf{3 0 0}$ & $\mathbf{1}$ & 0.60 & 1.50 \\
\hline Funcionamiento & $\mathbf{5 0 0}$ & 1.67 & $\mathbf{1}$ & 2.50 \\
\hline Cierre & $\mathbf{2 0 0}$ & 0.67 & 0.40 & $\mathbf{1}$ \\
\hline
\end{tabular}

Tabla 4. Asignación preliminar de las 1000 UIAP para las etapas del proyecto. Elaboración propia

Al realizar el cociente entre las 300 UIAP asignadas a la etapa de construcción $\left(c_{1}\right)$ y las 500 UIAP de la etapa de funcionamiento $\left(c_{2}\right)$, el valor obtenido $(0.60)$ corresponde a una importancia moderada de la etapa de funcionamiento respecto de la etapa de construcción.

En la celda correspondiente de la matriz de comparaciones por pares, presentada en la Tabla 5 , se le asignó el valor de preferencia recíproco $\left(C_{12}=1 / 3\right)$ según los valores de importancia presentados en la Tabla 1. El cociente entre las 300 UIAP de la etapa de construcción $\left(c_{1}\right)$ y las 200 UIAP de la etapa de cierre $\left(c_{3}\right)$ fue de 1.50, lo que indicó una preferencia moderada del criterio construcción con respecto al criterio cierre y en la celda $\left(C_{13}\right)$ de la Tabla 5 se asignó el valor 3 de la escala fundamental de la Tabla 1. El procedimiento se repitió hasta completar la valoración de todas las celdas.

\begin{tabular}{|c|c|c|c|}
\hline NIVEL 1 & Construcción & Funcionamiento & Cierre \\
\hline Construcción & $\mathbf{1}$ & $1 / 3$ & 3 \\
\hline Funcionamiento & 3 & $\mathbf{1}$ & 7 \\
\hline Cierre & $1 / 3$ & $1 / 7$ & 1 \\
\hline Sumas & $\mathbf{4 . 3 3}$ & $\mathbf{1 . 4 8}$ & $\mathbf{1 1 . 0 0}$ \\
\hline
\end{tabular}

Tabla 5. Matriz de comparaciones por pares de los criterios del nivel 1. Elaboración propia

Sumando de los valores de cada columna de la matriz de comparación por pares de la Tabla 5 y realizando el cociente entre el valor de cada celda y el valor de la suma de la columna correspondiente, se obtuvo la matriz normalizada presentada en la Tabla 6. A continuación, en una nueva columna, se sumaron las filas de la matriz de comparaciones por pares normalizada y luego se sumaron los valores de dicha columna. 


\begin{tabular}{|c|c|c|c|c|c|c|c|}
\hline NIVEL 1 & Construcción & Funcionamiento & Cierre & Suma & $\begin{array}{c}\text { Vector prioridad } \\
\left(\boldsymbol{p}_{\boldsymbol{j}}\right)\end{array}$ & $\begin{array}{c}\text { Vector } \\
\text { propio }\end{array}$ & Cociente \\
\hline Construcción & 0.23 & 0.23 & 0.27 & 0.73 & $\mathbf{0 . 2 4 3}$ & 0.73 & 3.01 \\
\hline Funcionamiento & 0.69 & 0.68 & 0.64 & 2.01 & $\mathbf{0 . 6 6 9}$ & 2.02 & 3.01 \\
\hline Cierre & 0.08 & 0.10 & 0.09 & 0.26 & $\mathbf{0 . 0 8 8}$ & 0.26 & 3.00 \\
\hline Sumas & $\mathbf{1}$ & $\mathbf{1}$ & $\mathbf{1}$ & $\mathbf{3 . 0 0}$ & $\mathbf{1}$ & $\mathbf{3 . 0 1}$ & \multicolumn{1}{c|}{} \\
\cline { 1 - 6 } & & & & &
\end{tabular}

Tabla 6. Matriz de comparación por pares normalizada para el nivel 1. Elaboración propia

Para obtener el coeficiente $\left(p_{j}\right)$, que corresponde al vector prioridad presentado en la Tabla 6, se realizó el cociente entre cada valor de la columna suma y el total de la suma. Se realizó el producto matricial entre la matriz de comparaciones por pares representada por la Tabla 5 y el vector prioridad $\left(p_{j}\right)$ de la Tabla 6 para obtener el principal vector propio y calcular el principal valor propio de la matriz $\lambda_{\max }=3.01$. En la última columna de la Tabla 6 se ubicaron los valores correspondientes al cociente entre los valores del vector propio y los del vector prioridad para cada fila de la matriz. La consistencia de la matriz se verificó calculando el Índice de consistencia aleatoria $I C=\left(\lambda_{\max }-3\right) /(3-1)=0.00$. Para obtener la relación de consistencia $(\mathrm{RC})$ se utilizó el Índice aleatorio, que depende del tamaño de la matriz, presentado en la Tabla $2, R C=I C /($ Índice aleatorio $)=0.000 .58=0.01 \mathrm{y}$ se verificó que los valores obtenidos resultaran inferiores al $10 \%$ recomendado por el autor del método (Saaty 1987, p. 172).

La cantidad de matrices de comparaciones por pares que se deben confeccionar es función del número de elementos incluidos en cada nivel de la estructura jerárquica del proyecto en estudio.

Para el proyecto minero del ejemplo analizado se necesitaron 44 matrices como se indica a continuación: Nivel 1 (Una matriz de comparación por pares para los criterios principales). Nivel 2 (Tres matrices de comparación por pares para los componentes). Nivel 3 (Dieciséis matrices de comparación por pares de actividades). Nivel 4 (Veinticuatro matrices de comparación por pares para las acciones). Para la confección de cada una de las 44 matrices necesarias se utilizó el método Delphi de convergencia (Landeta 1999, Conesa Fdez.Vítora 2010, p. 261-263).

En la Figura 2 se presenta la estructura jerárquica completa, con la distribución de las 1000 UIAP en cada uno de sus niveles, hasta llegar a las 32 acciones correspondientes a la Ingeniería básica del proyecto minero del ejemplo. 


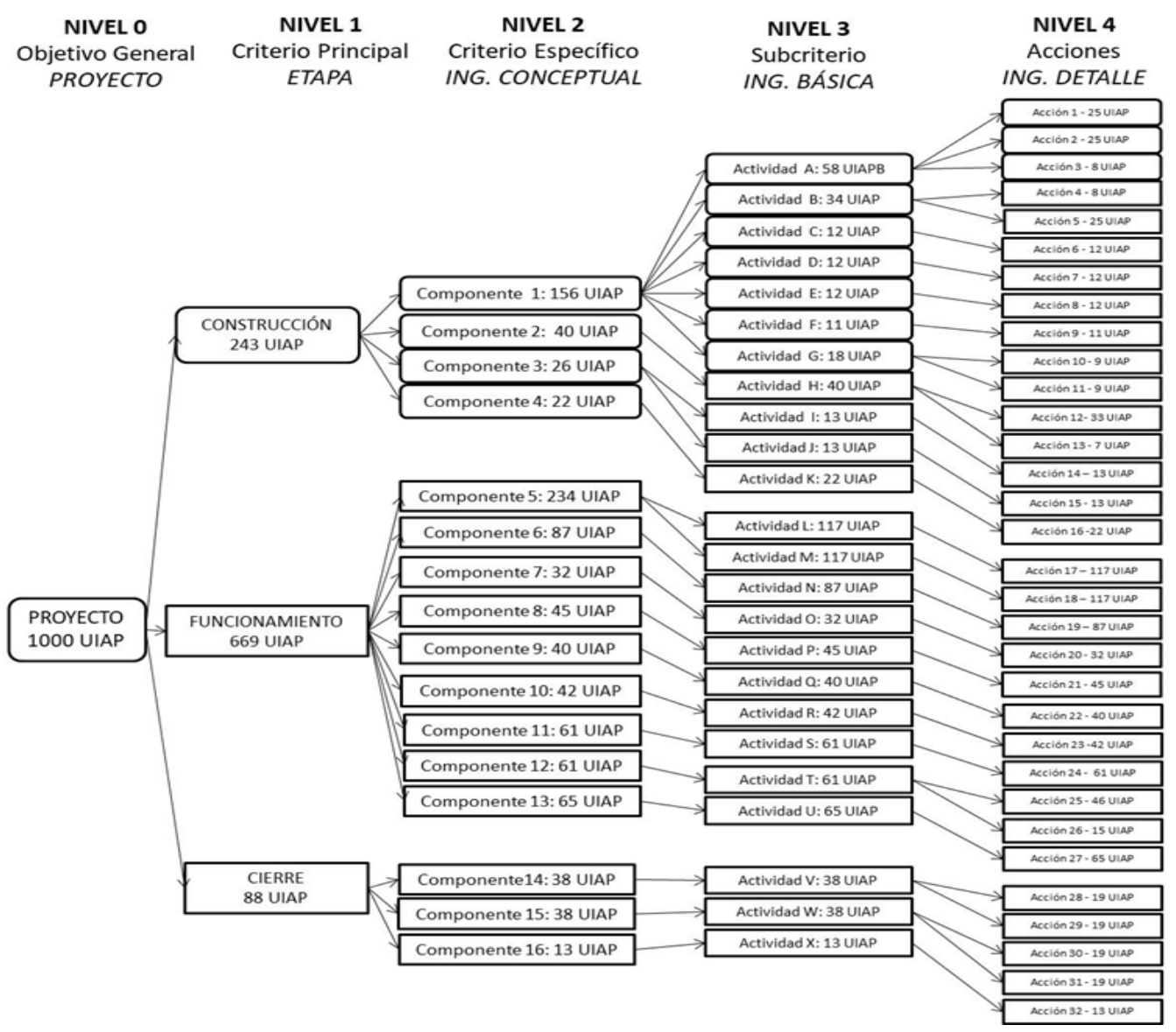

Figura 2. Estructura jerárquica del proyecto. Elaboración propia

Multiplicando por 1000 cada coeficiente $\left(p_{j}\right)$, calculado por aplicación del AHP, se obtuvieron los valores de las 32 UIAP correspondientes a la Ingeniería básica del proyecto del ejemplo que ocupan la última columna de la Tabla 7, en la que también se presentaron los valores de los coeficientes $\left(p_{j}\right)$ para las etapas, los componentes, las actividades y las acciones del proyecto.

Los 32 valores de las UIAP corresponden a las acciones del proyecto, son los que luego se ubican en cada columna ( $i$ ) de la matriz de EIA y complementan el cálculo de la importancia relativa de cada impacto ambiental identificado $\left(I_{i j}\right)$, al tener en cuenta los pesos relativos de los factores ambientales representados por las Unidades de Importancia de Parámetro (PIU: Parameter Importance Units) (Dee et al. 1972) y los pesos relativos de las acciones del proyecto a partir de los valores calculados para las UIAP. 


\begin{tabular}{|c|c|c|c|c|c|}
\hline $\begin{array}{c}\text { Coeficientes }(p j) \\
\text { Etapas }\end{array}$ & $\begin{array}{c}\text { Coeficientes }(p j) \\
\text { Componentes }\end{array}$ & \begin{tabular}{|c|}
$\begin{array}{c}\text { Coeficientes }(p j) \\
\text { Actividad }\end{array}$ \\
\end{tabular} & Acciones & $\begin{array}{c}\text { Coeficientes }(p j) \\
\text { Acciones }\end{array}$ & UIAP $(p j \times 1000)$ \\
\hline \multirow{16}{*}{$\begin{array}{c}\text { Construcción } \\
0,243\end{array}$} & \multirow{11}{*}{0,156} & \multirow{3}{*}{0,058} & ACCIÓN 1 & 0,025 & 25 \\
\hline & & & ACCIÓN 2 & 0,025 & 25 \\
\hline & & & ACCIÓN 3 & 0,008 & 8 \\
\hline & & \multirow{2}{*}{0,034} & ACCIÓN 4 & 0,008 & 8 \\
\hline & & & ACCIÓN 5 & 0,025 & 25 \\
\hline & & 0,012 & ACCIÓN 6 & 0,012 & 12 \\
\hline & & 0,012 & ACCIÓN 7 & 0,012 & 12 \\
\hline & & 0,012 & ACCIÓN 8 & 0,012 & 12 \\
\hline & & 0,011 & ACCIÓN 9 & 0,011 & 11 \\
\hline & & \multirow{2}{*}{0,018} & ACCIÓN 10 & 0,009 & 9 \\
\hline & & & ACCIÓN 11 & 0,009 & 9 \\
\hline & \multirow{2}{*}{0,04} & \multirow{2}{*}{0,04} & ACCIÓN 12 & 0,033 & 33 \\
\hline & & & ACCIÓN 13 & 0,007 & 7 \\
\hline & \multirow{2}{*}{0,026} & 0,013 & ACCIÓN 14 & 0,013 & 13 \\
\hline & & 0,013 & ACCIÓN 15 & 0,013 & 13 \\
\hline & 0,022 & 0,022 & ACCIÓN 16 & 0,022 & 22 \\
\hline \multirow{11}{*}{$\begin{array}{c}\text { Funcionamiento } \\
0,669\end{array}$} & \multirow{2}{*}{0,243} & 0,117 & ACCIÓN 17 & 0,117 & 117 \\
\hline & & 0,117 & ACCIÓN 18 & 0,117 & 117 \\
\hline & 0,087 & 0,087 & ACCIÓN 19 & 0,087 & 87 \\
\hline & 0,032 & 0,032 & ACCIÓN 20 & 0,032 & 32 \\
\hline & 0,045 & 0,045 & ACCIÓN 21 & 0,045 & 45 \\
\hline & 0,04 & 0,04 & ACCIÓN 22 & 0,040 & 40 \\
\hline & 0,042 & 0,042 & ACCIÓN 23 & 0,042 & 42 \\
\hline & 0,061 & 0,061 & ACCIÓN 24 & 0,061 & 61 \\
\hline & \multirow{2}{*}{0,061} & \multirow{2}{*}{0,061} & ACCIÓN 25 & 0,046 & 46 \\
\hline & & & ACCIÓN 26 & 0,015 & 15 \\
\hline & 0,065 & 0,065 & ACCIÓN 27 & 0,065 & 65 \\
\hline \multirow{5}{*}{$\begin{array}{c}\text { Cierre } \\
0,088\end{array}$} & \multirow{2}{*}{0,038} & \multirow{2}{*}{0,038} & ACCIÓN 28 & 0,019 & 19 \\
\hline & & & ACCIÓN 29 & 0,019 & 19 \\
\hline & \multirow{2}{*}{0,038} & \multirow{2}{*}{0,038} & ACCIÓN 30 & 0,019 & 19 \\
\hline & & & ACCIÓN 31 & 0,019 & 19 \\
\hline & 0,013 & 0,013 & ACCIÓN 32 & 0,013 & 13 \\
\hline
\end{tabular}

Tabla 7. Coeficientes $\left(p_{j}\right)$ de los elementos del proyecto. Elaboración propia

De la revisión de las UIAP presentadas en la Tabla 7 surge que la etapa de funcionamiento presenta las dos acciones con mayores valores (Acción 17: Excavar y Acción 18: Detonar, cada una con 117 UIAP). Con esos resultados se preparó una alternativa de proyecto para mejorar la etapa de funcionamiento, dado que es la de mayor duración y en la que se producen las dos acciones más importantes.

A partir de la revisión de la Ingeniería básica del proyecto se propusieron cambios operativos en la etapa de funcionamiento, para disminuir su duración de 10 a 8 años, a partir del aumento de la cantidad de equipos en la cantera, la combinación del uso de explosivos con excavación convencional para limitar emisiones atmosféricas, la reubicación de las instalaciones de procesamiento industrial para disminuir las distancias de transporte y la cantidad de vehículos necesarios, la reducción del consumo de combustibles fósiles con el uso de transporte sobre rieles, el aumento de la capacidad de las instalaciones industriales con la implementación de líneas de procesamiento paralelas, entre otros.

Con este nuevo escenario para la Ingeniería básica se reiteró la encuesta realizada a los 
paneles de expertos extraídos de los grupos sociales con interés en el proyecto, utilizando el método Delphi (Landeta 1999, Conesa Fdez.-Vítora 2010, p. 261-263), para reducir las 669 UIAP iniciales de la etapa de funcionamiento.

$\mathrm{Al}$ introducir los cambios mencionados en la Ingeniería básica, la nueva asignación preliminar de las 1000 UIAP entre los criterios del nivel 1 se estableció con los pesos relativos que se indican a continuación: Construcción (400 UIAP). Funcionamiento (400 UIAP). Cierre (200 UIAP).

Es decir que para la etapa de construcción la asignación se aumentó de 300 UIAP iniciales a 400 UIAP, la etapa de funcionamiento se redujo de 500 UIAP a 400 UIAP y la etapa de cierre conservó las 200 UIAP.

Con esta nueva asignación preliminar, se repitieron todos los cálculos necesarios, se verificó que la matriz de comparación por pares sea simétrica, recíproca y consistente y se obtuvieron los siguientes valores: Construcción (429 UIAP). Funcionamiento (429 UIAP). Cierre (142 UIAP).

Con esta nueva distribución preliminar, el índice de consistencia relativo $(C R=0.00)$ confirmó la validez del cálculo.

En la Tabla 8 se presentan los nuevos valores de los coeficientes $\left(p_{j}\right)$ para las etapas, los componentes, las actividades y las acciones del proyecto, después de analizar los cambios propuestos para la Ingeniería básica del proyecto. Los 32 valores de las UIAP para las acciones de la nueva alternativa de proyecto considerada, que se presentan en la última columna de la Tabla 8, son los que se ubican en las columnas (i) de la matriz de EIA para reiterar el cálculo de la importancia relativa de los impactos ambientales $\left(I_{i j}\right)$ ocasionados por las acciones del proyecto sobre los factores ambientales. 


\begin{tabular}{|c|c|c|c|c|c|}
\hline $\begin{array}{c}\text { Coeficientes (pj) } \\
\text { Etapas }\end{array}$ & $\begin{array}{c}\text { Coeficientes (pj) } \\
\text { Componentes }\end{array}$ & $\begin{array}{c}\text { Coeficientes (pj) } \\
\text { Actividad }\end{array}$ & Acciones & $\begin{array}{c}\text { Coeficientes (pj) } \\
\text { Acciones }\end{array}$ & UIAP (pj x 1000) \\
\hline \multirow{16}{*}{$\begin{array}{c}\text { Construcción } \\
0,429\end{array}$} & \multirow{11}{*}{0.247} & \multirow{3}{*}{0,091} & ACCIÓN 1 & 0,039 & 39 \\
\hline & & & ACCIÓN 2 & 0,039 & 39 \\
\hline & & & ACCIÓN 3 & 0,013 & 13 \\
\hline & & \multirow{2}{*}{0,053} & ACCIÓN 4 & 0,013 & 13 \\
\hline & & & ACCIÓN 5 & 0,040 & 40 \\
\hline & & 0,019 & ACCIÓN 6 & 0,019 & 19 \\
\hline & & 0,019 & ACCIÓN 7 & 0,019 & 19 \\
\hline & & 0,019 & ACCIÓN 8 & 0,019 & 19 \\
\hline & & 0,018 & ACCIÓN 9 & 0,018 & 18 \\
\hline & & \multirow{2}{*}{0,028} & ACCIÓN 10 & 0,014 & 14 \\
\hline & & & ACCIÓN 11 & 0,014 & 14 \\
\hline & \multirow{2}{*}{0.077} & \multirow{2}{*}{0,08} & ACCIÓN 12 & 0,064 & 64 \\
\hline & & & ACCIÓN 13 & 0,013 & 13 \\
\hline & \multirow{2}{*}{0.057} & 0,029 & ACCIÓN 14 & 0,029 & 29 \\
\hline & & 0,029 & ACCIÓN 15 & 0,029 & 29 \\
\hline & 0.047 & 0,047 & ACCIÓN 16 & 0,047 & 47 \\
\hline \multirow{11}{*}{$\begin{array}{c}\text { Funcionamiento } \\
0.429\end{array}$} & \multirow{2}{*}{0,129} & 0,064 & ACCIÓN 17 & 0,064 & 64 \\
\hline & & 0,064 & ACCIÓN 18 & 0,064 & 64 \\
\hline & 0,061 & 0,061 & ACCIÓN 19 & 0,061 & 61 \\
\hline & 0,024 & 0,024 & ACCIÓN 20 & 0,024 & 24 \\
\hline & 0,030 & 0,030 & ACCIÓN 21 & 0,030 & 30 \\
\hline & 0,026 & 0,026 & ACCIÓN 22 & 0,026 & 26 \\
\hline & 0,030 & 0,030 & ACCIÓN 23 & 0,030 & 30 \\
\hline & 0,042 & 0,042 & ACCIÓN 24 & 0,042 & 42 \\
\hline & \multirow{2}{*}{0,042} & \multirow{2}{*}{0,042} & ACCIÓN 25 & 0,032 & 32 \\
\hline & & & ACCIÓN 26 & 0,011 & 11 \\
\hline & 0,045 & 0,045 & ACCIÓN 27 & 0,045 & 45 \\
\hline \multirow{5}{*}{$\begin{array}{l}\text { Cierre } \\
0.143\end{array}$} & \multirow{2}{*}{0,061} & \multirow{2}{*}{0,061} & ACCIÓN 28 & 0,031 & 31 \\
\hline & & & ACCIÓN 29 & 0,031 & 31 \\
\hline & \multirow{2}{*}{0,061} & \multirow{2}{*}{0,061} & ACCIÓN 30 & 0,031 & 31 \\
\hline & & & ACCIÓN 31 & 0,031 & 31 \\
\hline & 0,020 & 0,020 & ACCIÓN 32 & 0,020 & 20 \\
\hline
\end{tabular}

Tabla 8. Coeficientes $\left(p_{j}\right)$ de los elementos para el proyecto reformulado. Elaboración propia

Como se mencionó previamente, las expresiones utilizadas en los métodos matriciales para el cálculo de la importancia relativa de los impactos ambientales $\left(I_{i j}\right)$ sólo tienen en cuenta la ponderación de los factores ambientales por medio de la asignación de las 1000 PIU (Parameter Importance Units). Esta valoración se presenta en el esquema de la Tabla 9 para la matriz de importancia de impactos.

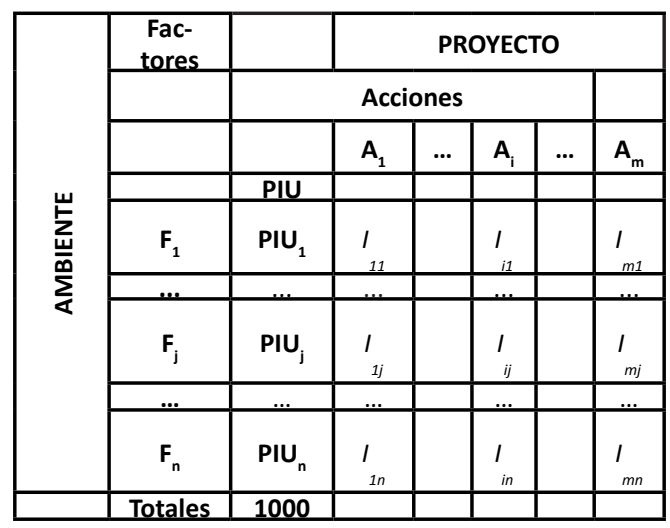

Tabla 9. Matriz de Importancia. Adaptado de: Conesa Fdez.-Vítora 1997, pág. 99 
En la matriz de importancia, representada en la Tabla 9, los valores absolutos y relativos se expresan en función de los valores correspondientes a las Unidades de Importancia de Parámetros (PIU: Parameter Importance Units) (Dee et al. 1972) según las siguientes expresiones:

Valor absoluto del impacto recibido por un factor $(\mathrm{j}): \quad I_{j}=\sum_{i}^{m} I_{i j}$

Valor relativo del impacto recibido por un factor $(\mathrm{j}): \quad I_{R j}=\frac{\sum_{i}^{m} I_{i j} * P I U_{j}}{\sum_{j}^{n} P I U_{j}}$

Valor absoluto del impacto causado por una acción (i): $I_{i}=\sum_{j}^{n} I_{i j}$

Valor relativo del impacto causado por una acción (i): $\quad I_{R i}=\frac{\sum_{j}^{n} I_{i j} * P I U_{j}}{\sum_{j}^{n} P I U_{j}}$

Con los valores de las 32 UIAP para las dos alternativas de proyecto consideradas, se confeccionaron las matrices de importancia. La Tabla 10 presenta el esquema de la matriz de importancia sobre los factores ambientales del medio físico, considerando la etapa de funcionamiento para la alternativa de proyecto.

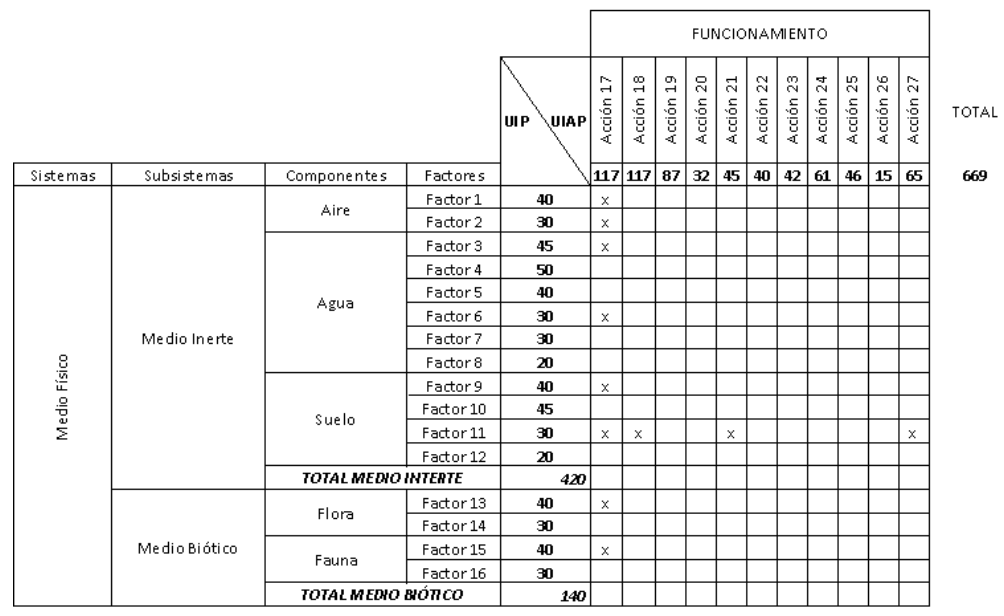

Tabla 10. Matriz de importancia para el funcionamiento de la alternativa inicial. Elaboración propia

Luego de implementar las modificaciones mencionadas a la Ingeniería básica del proyecto se preparó la Tabla 11 con los nuevos valores para los elementos del ambiente y para las acciones del proyecto. 


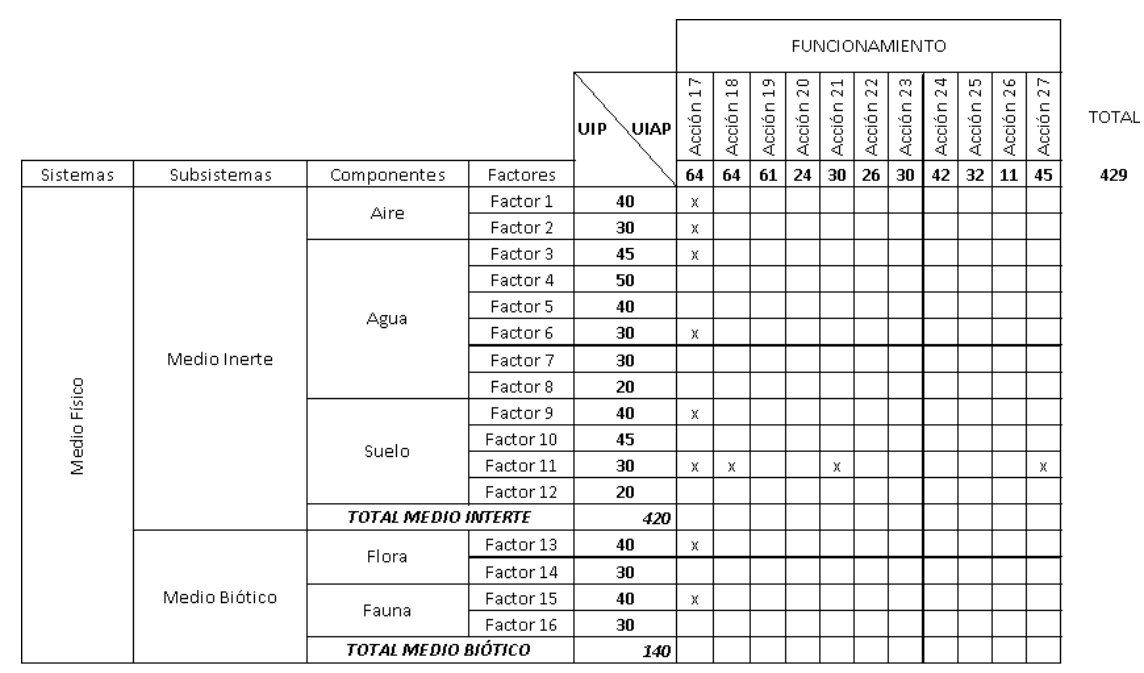

Tabla 11. Matriz de importancia para el funcionamiento de la alternativa modificada. Elaboración propia

Empleando el método descripto en las secciones previas, se incluyeron tanto las valoraciones relativas de las Unidades de Importancia de Parámetros (PIU: Parameter Importance Units) (Dee et al. 1972) como las de las Unidades de Importancia de Acciones de Proyecto (UIAP), utilizando las siguientes expresiones generales.

Valor absoluto del impacto recibido por un factor $\mathrm{j}: \quad I_{j}=\sum_{i}^{m} I_{i j}$

Valor relativo del impacto recibido por un factor $\mathrm{j}: \quad I_{R j}=\frac{P I U_{j} *\left[\sum_{i}^{m} I_{i j} * U I A P_{i}\right]}{\sum_{j}^{n} P I U_{j} * \sum_{i}^{n} U I A P_{i}}$

Valor absoluto del impacto causado por una acción i: $I_{i}=\sum_{j}^{n} I_{i j}$

Valor relativo del impacto causado por una acción i: $\quad I_{R i}=\frac{U I A P_{i} *\left[\sum_{j}^{n} I_{i j} * P I U_{j}\right]}{\sum_{j}^{n} \operatorname{PIU}_{j} * \sum_{i}^{n} U I A P_{i}}$

Sustituyendo en las expresiones (6) y (8) los valores de las PIU (Parameter Importance Units) y de las UIAP (Unidades de Importancia de Acciones de Proyecto), presentados en la Tabla 10. Matriz de importancia para la etapa de funcionamiento de la alternativa inicial, y tomando en todos los casos valores unitarios para los indicadores $\left(I_{i j}\right)$, se obtuvo el valor relativo del impacto ambiental recibido por el Factor $11 I_{R 11}=0.028$ y el valor relativo del impacto causado por la Acción 17 del proyecto $I_{R 17}=0.067$.

Reiterando la misma operación con los valores de la Tabla 11. Matriz de importancia para la etapa de funcionamiento de la alternativa modificada, el valor relativo obtenido para el impacto ambiental recibido por el Factor 11 resultó en $I_{R 11}=0.025$ y el 
valor relativo del impacto ambiental causado por la Acción 17 del proyecto fue $I_{R 17}=0.057$. Con los valores obtenidos para las UIAP de las dos alternativas de proyecto consideradas, la EIA se completa con la confección del programa de vigilancia y control con las medidas preventivas para las acciones más importantes y con la preparación del programa de monitoreo para el seguimiento de los factores ambientales más afectados. La definición de las medidas necesarias para el programa de vigilancia y control del proyecto se argumenta a partir de los valores relativos $\mathrm{I}_{R i}$ que permiten identificar las acciones más significativas. De la misma manera, para definir el programa de monitoreo de los factores ambientales más afectados, se utilizan los valores relativos $\mathrm{I}_{R j}$.

\section{Discusión}

Con la aplicación del procedimiento metodológico desarrollado a la Evaluación de Impacto Ambiental (EIA) para la Ingeniería básica del proyecto minero descripto, los resultados presentados permiten confirmar que se logró justificar matemáticamente la decisión de seleccionar una alternativa en particular.

La estructura jerárquica de cinco niveles propuesta, que representa al proyecto, las etapas, los componentes, las actividades y las acciones, es compatible con la desagregación del ambiente en subsistemas, componentes y factores utilizada para distribuir las 1000 PIU (Parameter Importance Units) (Dee et al. 1972) y con la estructura general de una matriz de impactos que ubica las acciones del proyecto en las columnas (i) y los factores ambientales en las filas (j) (Conesa Fdez.-Vítora 2010, pág. 229, Gómez Orea 2010, pág. 298).

El empleo del AHP para obtener los coeficientes (p_j) y distribuir 1000 UIAP (Unidades de Importancia de Acciones de Proyecto) entre los elementos del proyecto, implican una mejora en la descripción del proyecto y sus alternativas, permitiendo analizar la consistencia de las preferencias expresadas por los paneles de expertos extraídos de los grupos sociales con interés en el proyecto, utilizando el método Delphi (Landeta 1999, Conesa Fdez.-Vítora 2010, p. 261-263).

La caracterización del estado inicial de los elementos del ambiente no se ve afectada, puesto que el trabajo de los expertos en cada factor se complementa con una descripción más exhaustiva y mejor organizada de las acciones del proyecto y sus alternativas.

El estado inicial de los factores ambientales, determinado a partir de los estudios realizados por cada experto, queda representado en las filas ( $\mathrm{j}$ ) de la matriz de impactos por los valores de las PIU (Parameter Importance Units). Las acciones del proyecto ocupan las columnas (i) de la matriz de impactos donde se presenta el valor de la Unidad de Importancia de Acciones de Proyecto (UIAP) correspondiente.

Con las expresiones desarrolladas para calcular el valor relativo del impacto ambiental causado por las acciones del proyecto (I_Ri) y el valor relativo del impacto ambiental recibido por los factores (I_Rj), se obtiene la información necesaria para preparar el programa de vigilancia y control ambiental con las medidas preventivas para las acciones más significativas de la alternativa de proyecto seleccionada y el programa de monitoreo para el seguimiento de los factores ambientales más afectados.

Los grupos sociales con interés en el proyecto integran el panel de expertos que participan del proceso de asignación de pesos y de análisis de alternativas, utilizando encuestas tipo Delphi (Landeta 1999) que tienen en cuenta las preferencias de los participantes y con el empleo del proceso analítico jerárquico (AHP: Analytic Hierarchy Process) se obtienen valores numéricos para ordenarlas jerárquicamente. 


\section{Conclusiones}

El procedimiento metodológico desarrollado, aplicado a la EIA de un proyecto para la explotación de minerales metalíferos desarrollado a nivel de Ingeniería básica, desagregó el proyecto en una estructura jerárquica compuesta por cinco niveles, calculó los valores de los pesos relativos de todos sus elementos a partir de la obtención del conjunto de coeficientes (p_j) y los expresó en valores adimensionales de Unidades de Importancia de Acciones de Proyecto (UIAP).

Esta nueva forma de presentar la descripción de un proyecto y el análisis de sus alternativas, aplicable a otros tipos de proyectos, también incorporó la consulta a paneles de expertos extraídos de los grupos sociales con interés en su realización, participando en la asignación preliminar de pesos. El resultado de este procedimiento es un método que emplea el proceso analítico jerárquico como herramienta matemática de decisión multicriterio para analizar comparativamente los valores de las Unidades de Importancia de Acciones de Proyecto (UIAP) que se obtienen antes y después de la introducción de cambios en el proyecto.

Con esta innovación los tomadores de decisión analizan las etapas, los componentes, las actividades y las acciones de su proyecto para implementar los cambios necesarios en las diferentes fases de su desarrollo para identificar la mejor alternativa técnica, económica, social y ambiental que satisfaga los objetivos del proyecto.

Por otra parte las autoridades responsables por la habilitación de los proyectos sometidos al procedimiento de EIA pueden verificar con mayor objetividad si la alternativa seleccionada es la mejor de las analizadas y los potenciales afectados por la ejecución del proyecto también pueden participar en la discusión de las alternativas consideradas a partir del conocimiento de los elementos más significativos del proyecto. 


\section{Referencias}

Blanco Morón, A., Delgado Calvo-Flores, M., Martín Ramos, J. M., Polo Almohano, M. P. (2009). "AIEIA: Software for fuzzy environmental impact assessment". Expert Systems with Applications. 36 (5), 9135-9149. DOI: 10.1016/j.eswa.2008.12.055.

Canter, L. W. (1982). "Environmental Impact Assessment”. Impact Assessment, 1:2, 6-40, DOI: 10.1080/07349165.1982.9725447.

Canter, L. W., Canty, G. A. (1993). "Impact significance determination - Basic considerations and sequenced approach”. Environ Impact Assess Rev. 13 (5), 275-297. DOI: 10.1016/0195-9255(93)90020-C.

Cardenas, Ibsen, C., Halman, Johannes, I. M. (2016). "Coping with uncertainty in environmental impact assessments: Open techniques”. Environmental Impact Assessment Review. (60), 24-39. DOI: 10.1016/j.eiar.2016.02.006.

Cloquell-Ballester, V. A., Monterde-Díaz, R., Cloquell-Ballester, V. A., Santamarina-Siurana, M. C. (2007). "Systematic comparative and sensitivity analyses of additive and outranking techniques for supporting impact significance assessments". Environmental Impact Assessment Review. 27 (1), 62-83. DOI: 10.1016/j.eiar.2006.08.005.

Conesa Fdez.-Vítora, V. (1993). Guía Metodológica para la Elaboración de una Evaluación de Impacto Ambiental. MundiPrensa. Madrid. 284 pp.

Conesa Fdez.-Vítora, V. (2010). Guía Metodológica para la Elaboración de una Evaluación de Impacto Ambiental. 4a edición revisada y ampliada. MundiPrensa. Madrid. 864 pp.

Dee, N., Baker, J., Drobny, N., Duke, K., Whitman, I., Fahringer, D. (1972). An Environmental Evaluation System for Water Resource Planning. Bureau of Reclamation U.S. Department of the Interior. Battelle Columbus Laboratories. 9 (3), 523-535. DOI: 10.1029/WR009i003p00523.

Enea, M., Salemi, G. (2001). "Fuzzy approach to the environmental impact evaluation". Ecological Modelling. 136 (2-3), 131-147. DOI: 10.1016/S0304-3800(00)00380-X.

Garmendía, A., Salvador, A., Crespo, C., Garmendía, L. (2005). Evaluación de Impacto Ambiental. Pearson Educación S.A., Madrid. 416 pp.

Gómez Orea, D. (2010). Evaluación de Impacto Ambiental. Un instrumento preventivo para la gestión ambiental. $2^{\mathrm{a}}$ edición. Reimpresión. MundiPrensa. Madrid. 749 pp.

Goyal, S. K., Deshpande, V. A. (2001). "Comparison of weight assignment procedures in evaluation of environmental impacts". Environmental Impact Assessment Review. 21 (6), 553-563. DOI: 10.1016/S0195-9255(01)00086-5.

Hillier, Frederik S., Liebermann, Gerald J. (2010). Introducción a la investigación de operaciones. $9^{a}$ edición. McGraw-Hill. México D. F. 978 pp.

Hoyos, D. (2010). The state of the art of environmental valuation with discrete choice experiments. Ecological Economics. 69 (8), 1595-1603. DOI: 10.1016/j.ecolecon.2010.04.011. Huang, J. P., Poh, K. L., Ang, B. W. (1995). "Decision Analysis in Energy and Environmental Modeling”. Energy. 20, (9), 843-855. DOI: 10.1016/0360-5442(95)00036-G. 
Huang, Ivy B., Keisler, Jeffrey, Linkov, Igor. (2011). "Multi-criteria decision analysis in environmental sciences: Ten years of applications and trends". Science of the Total Environment. 409, (19), 3578-3594. DOI: 10.1016/j.scitotenv.2011.06.022.

Ijäs, A., Kuitunen, M. T., Jalava, K. (2010). "Developing the RIAM method (rapid impact assessment matrix) in the context of impact significance assessment”. Environmental Impact Assessment Review. 30 (2), 82-89. DOI: 10.1016/j.eiar.2009.05.009.

Janssen, R. (2001). "On the Use of Multi-Criteria Analysis in Environmental Impact Assessment in The Netherlands”. J. Multi-Crit. Decis. Anal. 10, (2), 101-109. DOI: 10.1002/ mcda.293.

Kaya, T., Kahraman, C. (2011). "An integrated fuzzy AHP-ELECTRE methodology for environmental impact assessment”. Expert Systems with Applications. 38 (7), 8553-8562. DOI: 10.1016/j.eswa.2011.01.057.

Laivina, L., Pubule, M, R. (2014). "A multi-factor approach to evaluate environmental impact statements". Agronomy Research, 12 (3), 967-976. http://agronomy.emu.ee/ vol123/2014_3_32_b5.pdf.

Landeta, Jon. (1999). El Método Delphi. Una Técnica de Previsión del Futuro. Ariel. Barcelona. $223 \mathrm{pp}$.

Leknes, E. (2001). "The roles of EIA in the decision-making process". Environmental Impact Assessment Review. 21 (4), 309-334. DOI: 10.1016/S0195-9255(00)00081-0.

Leopold, L., Clarke, F., Hanshaw, B. y Balsley, J. (1971). A Procedure for Evaluating Environmental Impact. U. S. Geological Survey Circular 645. United States Department of the Interior. Washington. DOI: 10.3133/circ645.

Lin, Y. H., Cheng, H. P., Tseng, M. L., Tsai, J. C. C. (2010). "Using QFD and ANP to analyze the environmental production requirements in linguistic preferences". Expert Systems with Applications. 37 (3), 2186-2196. DOI: 10.1016/j.eswa.2009.07.065.

Liu, K. F. R., Lai, J. H. (2009). "Decision-support for environmental impact assessment: A hybrid approach using fuzzy logic and fuzzy analytic network process”. Expert Systems with Applications. 36 (3-1), 5119-5136. DOI: 10.1016/j.eswa.2008.06.045.

Parashar, A., Paliwal, R., Rambabu, P. (1997). "Utility of Fuzzy Cross-Impact Simulation in Environmental Assessment”. Environ Impact Assess Rev. 17 (6), 427-447. DOI: 10.1016/ S0195-9255(97)00046-2.

Peche, R., Rodríguez, E. (2011). "Environmental impact assessment by means of a procedure based on fuzzy logic: A practical application”. Environmental Impact Assessment Review. 31 (2), 87-96. DOI: 10.1016/j.eiar.2010.03.006.

Perdicoúlis, A., Glasson, J. (2006). Causal networks in EIA. Environmental Impact Assessment Review. 26 (6), 553-569. DOI: 10.1016/j.eiar.2006.04.004.

Peterson. G. L., Gemmell, R. S., \& Schofer, J. L. (1974). "Assessment of environmental impacts. Multidisciplinary judgments of large scale projects”. EKISTICS. 37 (218), 23-30.

Ramanathan, R. (2001). "A note on the use of the analytic hierarchy process for environmental impact assessment". Journal of Environmental Management. 63, (1), 27-35. DOI:10.1006/jema.2001.0455. 
Saaty, T. L. (1977). "A scaling method for priorities in hierarchical structures". Journal of Mathematical Psychology. 15 (3), 234-281. DOI: 10.1016/0022-2496(77)90033-5.

Saaty, T. L. (1978). "Modeling Unstructured Decision Problems - The Theory of Analytical Hierarchies”. Mathematics and Computers in Simulation. 20 (3), 147-158. DOI: 10.1016/0378-4754(78)90064-2.

Saaty, R. W. (1987). “The Analytic Hierarchy Process-What it is and how it to used". Mathematical Modelling. 9 (3-5), 161-176. DOI: 10.1016/0270-0255(87)90473-8.

Saaty, T. L. (2008). "Decision making with the analytic hierarchy process". Int. J. Services Sciences. 1 (1), 83-98. DOI: 10.1504/IJSSCI.2008.017590.

Steinemann, A. (2001). "Improving alternatives for environmental impact assessment". Environmental Impact Assessment Review. 21 (1), 3-21. DOI: 10.1016/S0195-9255(00)000755.

Taha, H. A. (2006). Operations research: an introduction. 8th ed. Pearson. Prentice Hall. U.S.A. 813 pp.

Thompson, M. A. (1990). "Determining impact significance in EIA: a review of 24 methodologies". Journal of Environmental Management. 30 (3), 235-250. DOI: 10.1016/03014797(90)90004-G.

Toro, J., Requena, I., Duarte, O., Zamorano, M. (2013). "A qualitative method proposal to improve environmental impact assessment". Environmental Impact Assessment Review. 43, 9-20. DOI: 10.1016/j.eiar.2013.04.004.

van der Werf, H. M. G., Tzilivakis, J., Lewis, K., Basset-Mens, C. (2007). Environmental impacts of farm scenarios according to five assessment methods. Agriculture, Ecosystems and Environment. 118 (1-4), 327-338. DOI: 10.1016/j.agee.2006.06.005.

Warner, M. L., Preston, E. H. (1974). A Review of Environmental Impact Assessment Methodologies. EPA-600/5-74-002. Office of Reasearch and Development. U.S.

Environmental Protection Agency. Washington, D.C. 20460. 36 pp.

Yang, M., Khan, F. I., Sadiq, R. (2011). "Prioritization of environmental issues in offshore oil and gas operations: A hybrid approach using fuzzy inference system and fuzzy analytic hierarchy process". Process Safety and Environmental Protection. 89 (1), 22-34. DOI: 10.1016/j.psep.2010.08.006.

Zhao, M. Y., Cheng, C. T., Chau, K. W., Li, G. (2006). "Multiple criteria data envelopment analysis for full ranking units associated to environment impact assessment”. International Journal of Environment and Pollution. 28 (3-4), 448-464. DOI: 10.1504/IJEP.2006.011222. 